\title{
Analgesic efficacy of imrecoxib for postoperative pain following oral surgery: a prospective randomized, active-controlled, non-inferiority trial
}

\author{
Yu Jiang ${ }^{1,2}$, Lili $\mathbf{Q i}^{1,2}$, Jie Liu ${ }^{1,2}$, Heming $\mathrm{Wu}^{1,2}$, Yi $\mathrm{Li}^{3}$, Linhu Zou ${ }^{3}$, Zhiwei Guo ${ }^{3}$, Jinqiang Wang ${ }^{3}$, \\ Huaiqi $\mathrm{Li}^{1,2}$
}

${ }^{1}$ Jiangsu Key Laboratory of Oral Diseases, Nanjing Medical University, Nanjing, China; ${ }^{2}$ Department of Oral and Maxillofacial Surgery, Affiliated Hospital of Stomatology, Nanjing Medical University, Nanjing, China; Jiangsu Hengrui Pharmaceutical Co., Ltd., Lianyungang, China

Contributions: (I) Conception and design: H Li, J Liu, H Wu, Y Li; (II) Administrative support: H Li; (III) Provision of study drug and randomized tool: L Zou, Z Guo, J Wang; (IV) Drug management and pain assessment: L Qi, J Liu; (V) Collection and assembly of data: H Li, Y Jiang; (VI) Data analysis and interpretation: H Li, Y Jiang; (VII) Manuscript writing: All authors; (VIII) Final approval of manuscript: All authors.

Correspondence to: Huaiqi Li. Department of Oral and Maxillofacial Surgery, Affiliated Hospital of Stomatology, Nanjing Medical University, Nanjing 210029, China. Email: lihuaiqi781214@sohu.com.

Background: Imrecoxib, a novel cyclooxygenase (COX-2) selective non-steroidal anti-inflammatory drug (NSAID), has been approved in China for more than 9 years. This study aimed to assess the efficacy and safety of imrecoxib compared with celecoxib for patients with moderate or severe acute pain following oral surgery.

Methods: Patients with moderate or severe pain within 6 hours following surgery were enrolled in this randomized, active-control trial. Patients were randomly assigned (1:1) to receive either imrecoxib or celecoxib. Pain assessments on the visual analog scale, verbal rating scale, and pain relief were conducted at $0.5,1,2,4,6,9,12$, and 24 hours after the first dose. Adverse events were also recorded.

Results: Eighty-seven patients were approached from November 2018 to August 2019. Of these, 60 were eligible for randomization. Ultimately, 56 patients (imrecoxib group, $n=27$; celecoxib group, $n=29$ ) were included in the analysis. The difference in total pain relief (TOTPAR) between the imrecoxib and celecoxib groups was 1.03 [95\% confidence interval (CI): $-1.31-3.77$ ], with the lower bound of the CI above the specified non-inferiority boundary. No perioperative complications were observed in the imrecoxib group during the 24-hour period after the first dose.

Conclusions: Imrecoxib could significantly relieve pain and has a non-inferior analgesic efficacy compared to celecoxib with good tolerance following oral surgery.

Keywords: Non-steroidal anti-inflammatory drugs (NSAIDs); oral surgery; postoperative pain

Submitted Dec 23, 2020. Accepted for publication Feb 24, 2021.

doi: $10.21037 /$ atm-21-264

View this article at: http://dx.doi.org/10.21037/atm-21-264

\section{Introduction}

Postoperative acute pain management is one of the essential elements of enhanced recovery after surgery (ERAS) (1). In some cases, it may result in harmful physiological and psychological consequences, which may delay recovery and the return to normal daily functioning. Most importantly, it has been recognized that inadequate postoperative pain treatment leads to chronic pain (2). Approximately 50-80\% of patients suffer moderate to severe pain following oral surgery, which usually peaks within 3 to 6 hours after surgery $(3,4)$. Non-steroidal anti-inflammatory drugs (NSAIDs) are widely recommended in postoperative pain therapy to relieve postoperative pain by adding/substituting opioid-containing analgesic combinations (5). However, conventional NSAIDs 
have been reported to be associated with impaired platelet function and gastrointestinal adverse events (AEs) $(6,7)$. Imrecoxib, a novel cyclooxygenase-2 (COX-2) selective NSAID (8), has been approved in China for more than 9 years. It has been shown to relieve osteoarthritis pain as effectively as celecoxib, and also has excellent gastrointestinal tolerability (9). One randomized trial demonstrated that imrecoxib could also achieve a good postoperative analgesic effect compared to the placebo following spinal surgery by being used in a pre-emptive fashion (10). Therefore, imrecoxib might be a good selective NSAID option for the treatment of acute pain following oral surgery.

The aim of this prospective randomized, activecontrolled, non-inferiority trial was to assess the efficacy and safety of imrecoxib for treating moderate or severe acute pain following oral surgery. We hypothesized that imrecoxib would reduce pain scores and exhibit non-inferior pain-relief compared with celecoxib for moderate or severe postoperative pain. We present the following article in accordance with the CONSORT reporting checklist (available at http://dx.doi.org/10.21037/atm-21-264).

\section{Methods}

\section{Patients}

This prospective randomized, active-controlled trial was performed in the wards of the Affiliated Stemmatological Hospital of Nanjing Medical University in China. Patients aged between 18 and 65 years old were eligible if they suffered moderate or severe pain as defined by a visual analogue scale (VAS) score $\geq 40 \mathrm{~mm}$ within 6 hours following oral surgery. Oral surgery included the extraction of an impacted mandibular molar or maxillary sinus cyst, which are two of the most common oral surgeries. Patients with an allergy to sulfa-based drugs, those with prior adverse reaction to NSAIDs, or those who were on chronic narcotics alternative pain management regimens prior to surgery were excluded.

\section{Randomization and interventions}

After confirmation of eligibility, patients were randomized (1:1) to receive either imrecoxib or celecoxib using the simple randomization method. The allocation sequence was computer-generated (http://tools.medsci.cn/rand/ getNewNum).

Patients received either $400 \mathrm{mg}$ imrecoxib (Jiangsu Hengrui
Pharmaceutical Co., Ltd., Lianyungang, China) or $400 \mathrm{mg}$ celecoxib (Pfizer Inc., New York, America). Pain assessments were conducted at $0.5,1,2,4,6,9,12$, and 24 hours after the first dose. If needed, patients could receive an additional $200 \mathrm{mg}$ imrecoxib or $200 \mathrm{mg}$ celecoxib no sooner than 2 hours after the first dose, and in these cases, pain assessments were conducted at 0 and 2 hours after the second dose and 24 hours after the first dose. Rescue medications were allowed if the patients still suffered from intolerable pain.

\section{Ethical statement}

This trial was approved by the ethics committee of the Stemmatological Hospital of Nanjing Medical University in China (ethics committee reference number YJ2018009-02). All procedures performed in this study involving human participants were in accordance with the Declaration of Helsinki (as revised in 2013). All participants provided written informed consent before enrollment.

\section{Assessments and endpoints}

Postoperative pain was measured by the VAS, the verbal rating scale (VRS), and pain relief (PR) at each time point. Overall assessment of the treatment was conducted at 24 hours. VAS assessment was performed using a nongraduated 100-mm VAS, which was labeled "no pain" at the left pole and "worst pain imaginable" at the right pole. Patients marked their current pain level on the scale directly. VRS assessment was performed using a 4-point scale where $0=$ 'no pain', 1 = 'mild pain', 2 = 'moderate pain', 3 = 'severe pain' or 'excruciating pain or agony'. PR assessment was performed using a 5 -point scale where $0=$ 'no pain relief', $1=$ 'mild pain relief', 2 = 'partial pain relief', 3 = 'perceptible pain relief', or $4=$ 'complete pain relief'. Overall assessment of the treatment was performed using a 4-point scale where $0=$ 'poor', 1 = 'fair', 2 = 'good', or 3 = 'excellent'.

Total pain relief (TOTPAR) was defined as the sum of the PR score at each time point over a 24-hour post-dosing period. The pain intensity difference (PID) was defined as a VAS score difference from the baseline. The sum of pain intensity difference (SPID) was defined as the sum of the PID at each time point over a 24-hour post-dosing period. Physical examinations and laboratory observations were conducted at baseline and on the following morning. AEs were recorded during the 24-hour period after the first dose.

The primary endpoint of the study was TOTPAR, and the secondary endpoints included PID, PR at each time 


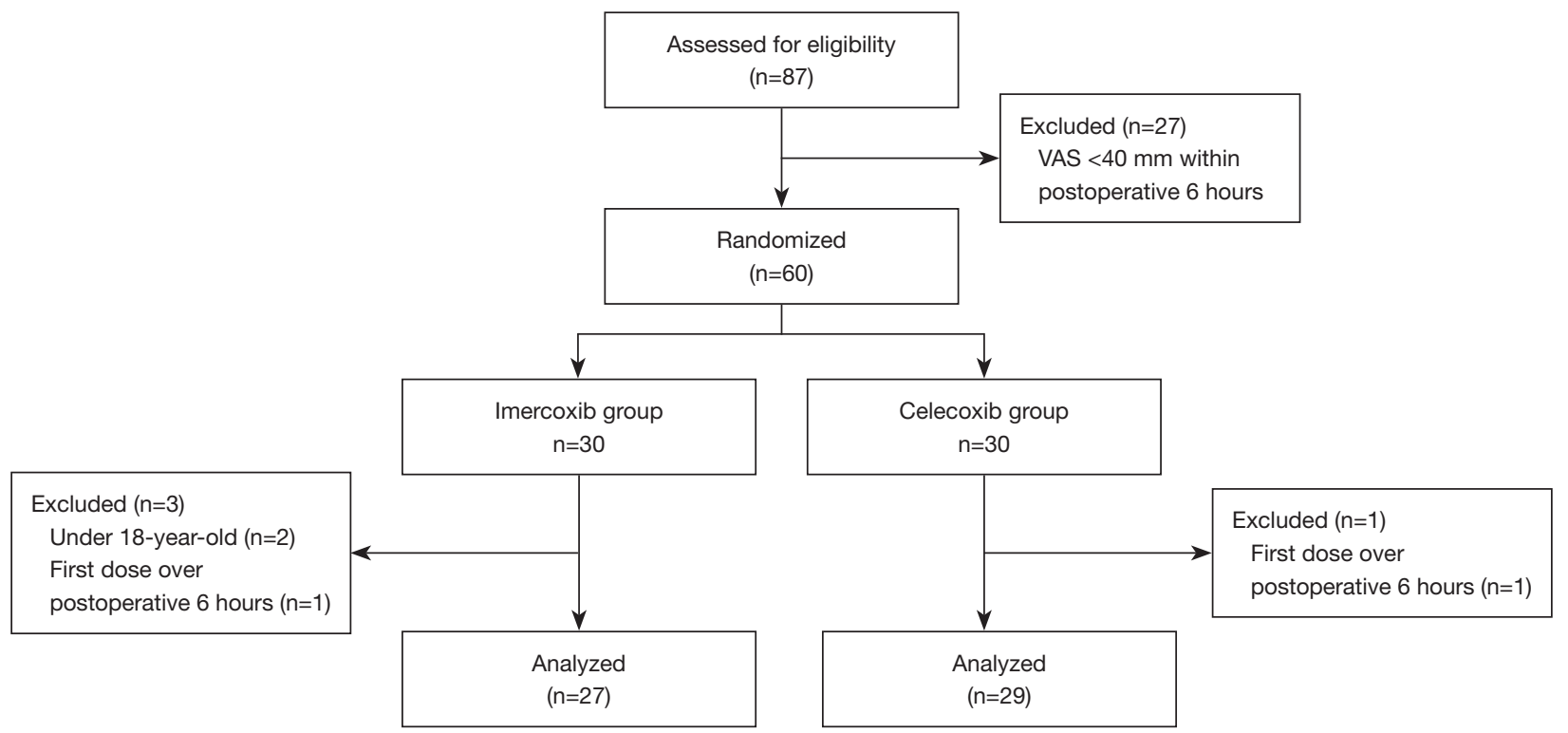

Figure 1 Flow chart of this trial.

point, SPID, overall assessment, rescue medicine, and AEs.

\section{Surgery}

All oral surgeries were performed by the same surgeon (Dr. Huaiqi Li) and followed the same anesthetic protocol during the operation. Operations were scheduled before 12 am to ensure that the pain assessments would not be conducted during the middle of the night. Other anesthetic properties were forbidden during the study and drug combinations were recorded.

\section{Sample size}

The sample size calculation for the two-tailed testing of the imrecoxib non-inferior hypothesis was based on the TOTPAR. The TOTPAR was estimated as $13 \pm 4.5$ [mean \pm standard deviation (SD)] in both groups. A non-inferiority margin of -3 was chosen as it represents approximately half of the observed TOTPAR of the placebo based on the placebo-control celecoxib trail (11). According to our power analysis $(\alpha=0.05, \beta=0.2)$, a sample size of 30 participants per group was required with $10 \%$ dropout rate.

\section{Statistical analysis}

SAS 9.4 (North Carolina State University, America) was used for statistical analysis. Parametric variables were reported as mean $\pm \mathrm{SD}$ and were analyzed between the groups using the independent-sample t-test. Nonparametric variables were reported as median [interquartile range (IQR)] and were compared between both groups using the Mann-Whitney U test. Categorical variables were reported as a number (proportion) and were evaluated using Fisher's exact or chi squared $\left(\chi^{2}\right)$ tests where appropriate. All of the reported $\mathrm{P}$ values were two-tailed, and a $\mathrm{P}$ value of less than 0.05 was considered statistically significant.

\section{Results}

Eighty-seven patients were approached between November 2018 and August 2019. Of these, 60 patients were randomized (1:1) to receive either imrecoxib or celecoxib. Two patients withdrew from the imrecoxib group as they were under the age of 18 years. Finally, 56 patients (imrecoxib group, $n=27$; celecoxib group, $n=29$ ) were included in the analysis. Only one patient in the celecoxib group had an additional $200 \mathrm{mg}$ celecoxib treatment (Figure 1). There were no significant differences in the characteristics at baseline between the two groups (all $\mathrm{P}>0.05$ ) (Table 1). The mean ages of patients in the imrecoxib and celecoxib groups were $30.07 \pm 12.13$ and $33.14 \pm 10.39$ years, respectively. The two groups were well matched in terms of surgical levels, surgical times, and baseline pain. Postoperative pain by VAS was balanced separately, with the average VAS of patients in the imrecoxib and celecoxib groups being $57.41 \pm 11.88$ 
Table 1 Demographic and baseline characteristics of the included patients

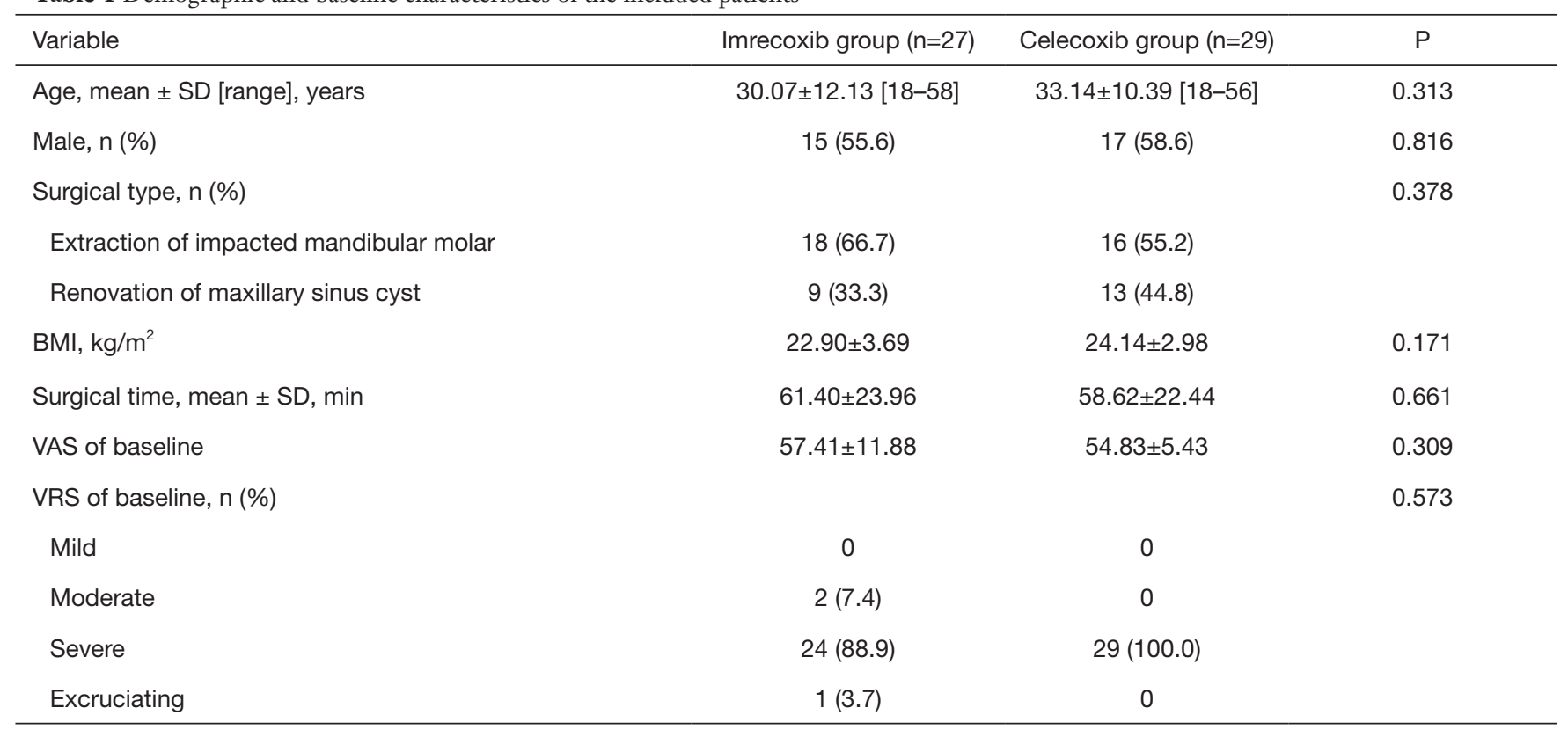

SD, standard devitation; BMI, body mass index; VAS, visual analog scale; VRS, verbal rating scale.

and $54.83 \pm 5.43 \mathrm{~mm}$, respectively. In the imrecoxib group, 2 (7.4\%) patients suffered moderate pain, 24 (88.9\%) suffered severe pain, and $1(3.7 \%)$ suffered excruciating pain, while all 29 patients $(100 \%)$ in the celecoxib group suffered severe pain.

\section{Primary endpoint}

The mean TOTPAR during the 24-hour period postdosing was not significantly different between the two groups $(10.44 \pm 5.54$ in the imrecoxib group vs. $9.52 \pm 3.38$ in the celecoxib group, $\mathrm{P}=0.457$ ) (Table 2). The difference in TOTPAR for imrecoxib compared with celecoxib was 1.03 [95\% confidence interval (CI): $-1.31-3.77$ ], with the lower bound of the $\mathrm{CI}$ above the specified non-inferiority boundary (Figure 2A).

\section{Secondary endpoints}

In general, no significant difference was observed in PR, PID, and SPID between the two groups. Differences in the average $\mathrm{PR}$ at each time point over the 24 -hour postdosing period were negligible (all $\mathrm{P}>0.05$ ). The PR peaked at 4 hours in both groups (Figure $2 B$ ). The mean PID scores of both groups increased notably over time, and the mean PID scores of the imrecoxib group were comparable to those of celecoxib group at each time point over the 24-hour post-dosing period (all $\mathrm{P}>0.05$ ) (Figure $3 A$ ). The mean SPID scores were not significantly different between the two groups $(208.27 \pm 63.20$ in the imrecoxib group $v s$. $200.86 \pm 40.80$ in the celecoxib group, $\mathrm{P}=0.612$ ) (Table 2). Also, the proportions of four point scale according to the VRS with imrecoxib were similar to those of celecoxib at each time point (all $\mathrm{P}>0.05$ ) (Figure $3 B$ ). All patients in both groups gave a good or excellent assessment and there was no considerable difference in the overall assessment between the two groups $(\mathrm{P}=0.186)$. None of the patients received rescue medicine during the study. There were no perioperative complications observed in either of the groups. Monitored complications included cardiovascular thrombotic events, myocardial infarction, stroke, and severe gastrointestinal upset. No perioperative complications were reported in both groups.

\section{Discussion}

In this prospective, randomized, active-controlled, noninferiority trial, imrecoxib showed a significant analgesic effect on moderate or severe acute pain therapy following oral surgery. No significant differences were found in TOTPAR, PID, PR at each time point, SPID, overall assessment, and rescue medicine between the imrecoxib 
Table 2 Comparison of pain intensity and other variables between the two groups

\begin{tabular}{lccc}
\hline Variable & Imrecoxib group $(\mathrm{n}=27)$ & Celecoxib group $(\mathrm{n}=29)$ & $\mathrm{P}$ \\
\hline TOTPAR, mean $\pm \mathrm{SD}$ & $10.44 \pm 5.54$ & $9.52 \pm 3.38$ & 0.457 \\
SPID, mean $\pm \mathrm{SD}, \mathrm{mm}$ & $208.27 \pm 63.20$ & $200.86 \pm 40.80$ & 0.612 \\
Overall assessment, $\mathrm{n}(\%)$ & & & 0.186 \\
Poor & 0 & 0 \\
Fair & 0 & $28(96.6)$ \\
Good & $23(85.2)$ & $1(3.4)$ \\
Excellent & $4(14.8)$ & 0 \\
Rescue medicine & 0 & 1 \\
\hline
\end{tabular}

TOTPAR, total pain relief; SD, standard deviation.
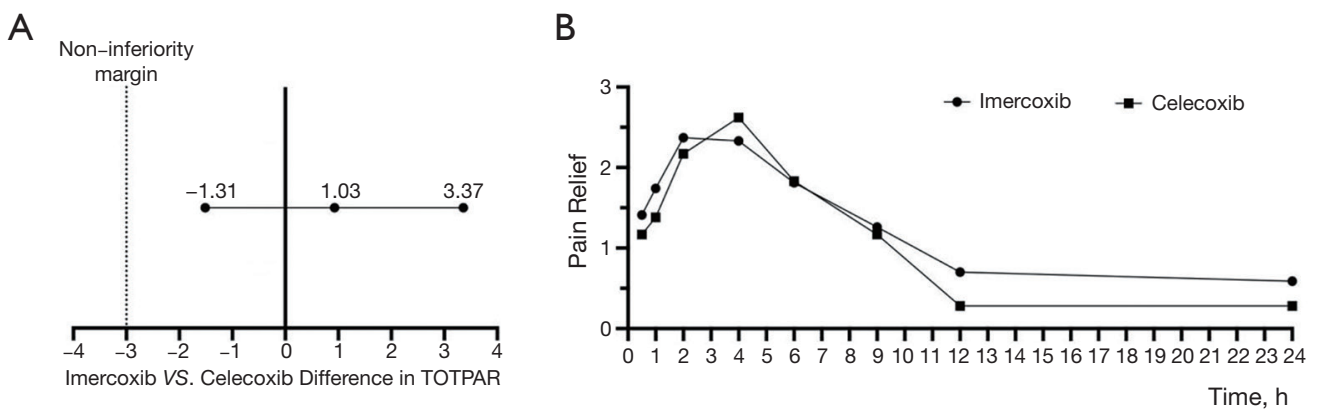

Figure 2 The total pain relief (TOTPAR) and pain relief (PR) results. (A) TOTPAR difference between the imrecoxib and celecoxib groups; and (B) mean RP over the 24-hour post-dosing period. The dotted line represents the -3 non-inferiority margin and the error bars represent $95 \%$ confidence interval (CI). The TOTPAR of the two groups are shown in Table 2.

and celecoxib groups (all $\mathrm{P}>0.005$ ). No perioperative complications were reported in both groups.

Considering that imrecoxib is currently only sold in China, most of the analgesic research on imrecoxib have been published in Chinese journals. To our knowledge, this is the first published trial comparing imrecoxib and celecoxib in the postoperative treatment of pain following oral surgery. For the primary endpoint, the non-inferiority of TOTPAR for imrecoxib compared with celecoxib was achieved. The similar analgesic efficacy in the imrecoxib and celecoxib groups was unsurprising, especially considering the mechanism of action of imrecoxib as well as the results of previous studies. Firstly, COX is mainly divided into COX-1 and COX-2. As a novel and moderately selective COX-2 inhibitor (COXIB), imrecoxib produces anti-inflammatory and anti-pain effects by inhibiting the expression of COX-2 messenger ribonucleic acid (mRNA) (8). In vitro experiments have shown that imrecoxib has a selective inhibitory effect on COX-1 and COX-2, with the inhibitory effect on COX-2 stronger than COX-1. In various guidelines for ERAS $(12,13)$, COXIBs and NSAIDs have been proposed as basic postoperative analgesic components, which can reduce the need for opioids. Selective COXIBs have been shown to be efficacious for dental pain management (14-16). Secondly, it has also been demonstrated that PR of ground walking at 12 weeks (the primary endpoint) was similar for those receiving imrecoxib and celecoxib in a phase 3 multi-center randomized blind-controlled trail study of imrecoxib in osteoarthritis (9). Another randomized controlled trial in axial spondylarthritis (axSpA) showed that patients experienced significant improvement in disease activity, functional parameters, and inflammatory markers when treated with imrecoxib for 12 weeks, and the efficacy of imrecoxib was not inferior to celecoxib (17). The present study provided support for the potential therapeutic effect 
A

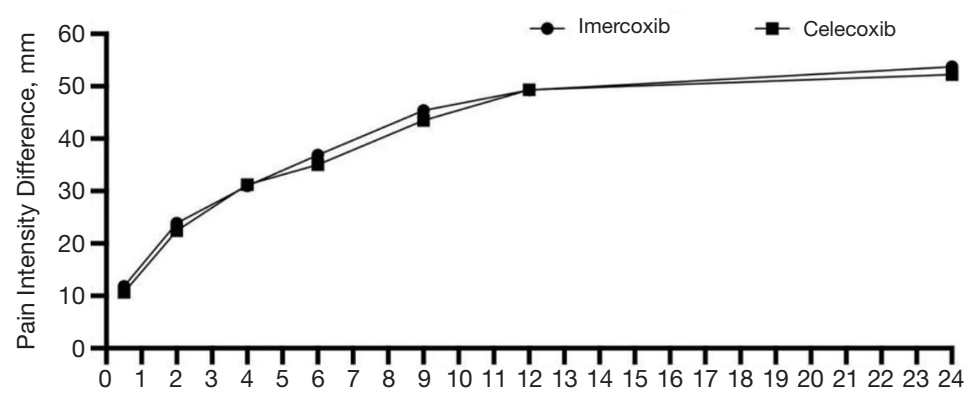

B
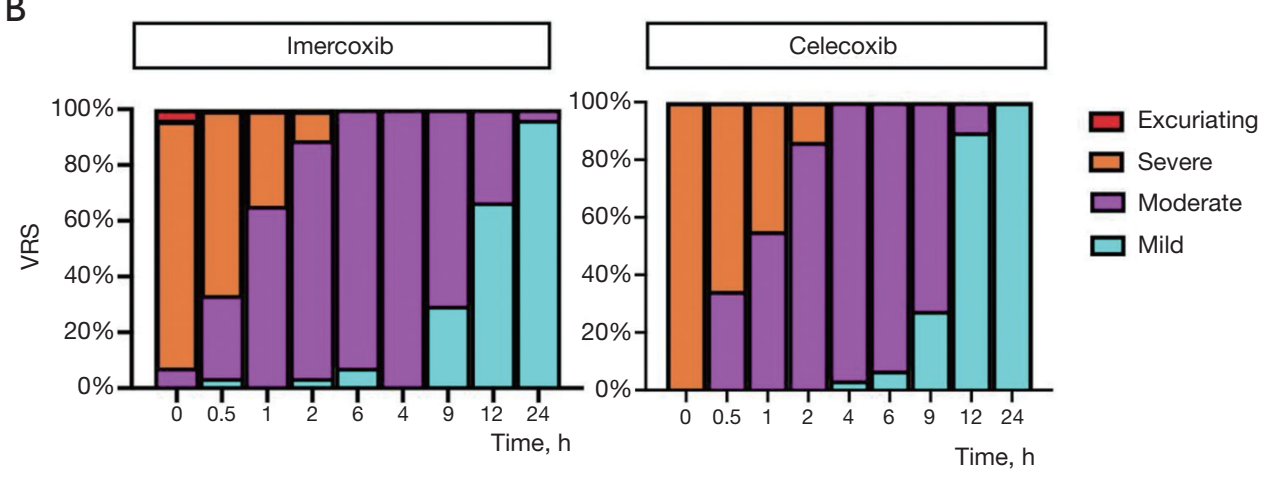

Figure 3 The pain intensity difference (PID) and verbal rating scale (VRS) results. (A) PID over 24 hours, mm; (B) VRS over 24 hours, \%.

of imrecoxib for acute postoperative pain, especially for acute pain following oral surgery.

No perioperative complications were reported in both groups during the 24-hour period after the first dose, which suggests a good tolerance of imrecoxib. In a phase 3 study, 351 osteoarthritis $(\mathrm{OA})$ patients aged $56.44 \pm 7.08$ years received imrecoxib $100 \mathrm{mg}$ twice per day and 118 patients aged $56.14 \pm 7.63$ years received celecoxib $200 \mathrm{mg}$ once per day for 8 weeks; the results showed no significant differences in AEs between the two groups $(7.85 \%$ in the imrecoxib group vs. $10.26 \%$ in the celecoxib group) (9). A multi-centered study involving 2,413 patients treated with imrecoxib for 8 weeks also showed a low AE profile for imrecoxib (18). Imrecoxib might have a better tolerance with a shorter treatment course in acute pain than that in OA.

The present study has several strengths. Firstly, the patient population was more representative for oral surgery, as patients undergoing extraction of an impacted mandibular molar or maxillary sinus cyst were both included, whereas other trials only included extractions of impacted mandibular molars. Secondly, we developed a good schedule plan to ensure the quality and feasibility of our assessments. Pain scores that are assessed frequently might disturb the patients' sleep, thereby leading slightly inaccurate results. Therefore, operations in this study were scheduled before 12 am to ensure that the pain assessments would not be conducted during the middle of the night. Finally, all surgeries were performed by the same surgeon and followed the same anesthetic protocol, which helped to reduce the two confounding factors affecting postoperative pain.

However, this trial does have some limitations that should be noted. As this study was designed as a singlecenter trial, it might not have the generalizability that a multi-center trial typically retains. Also, this study only recorded the AEs that occurred during first 24 hours after the first dose, which might be too short for a sufficient safety assessment. The use of imrecoxib for the treatment of long-term postoperative pain and functional recovery could be assessed in future trials. Advanced studies of imrecoxib are still needed for exploring the efficacy and safety of postoperative pain management.

In conclusion, this trial demonstrated that imrecoxib could significantly relieve postoperative pain. It was shown to have a non-inferior analgesic efficacy to celecoxib with a good tolerance following oral surgery. 


\section{Acknowledgments}

Funding: The study was supported by research grants from A Project Funded by the Priority Academic Program Development of Jiangsu Higher Education Institutions (PAPD, 2018-87).

\section{Footnote}

Reporting Checklist: The authors have completed the CONSORT reporting checklist. Available at http://dx.doi. org/10.21037/atm-21-264

Data Sharing Statement: Available at http://dx.doi. org/10.21037/atm-21-264

Conflicts of Interest: All authors have completed the ICMJE uniform disclosure form (available at http://dx.doi. org/10.21037/atm-21-264). The authors have no conflicts of interest to declare.

Ethical Statement: The authors are accountable for all aspects of the work in ensuring that questions related to the accuracy or integrity of any part of the work are appropriately investigated and resolved. This trial was approved by the ethics committee of the Stemmatological Hospital of Nanjing Medical University in China (ethics committee reference number YJ2018-009-02). All procedures performed in this study involving human participants were in accordance with the Declaration of Helsinki (as revised in 2013). All participants provided written informed consent before enrollment.

Open Access Statement: This is an Open Access article distributed in accordance with the Creative Commons Attribution-NonCommercial-NoDerivs 4.0 International License (CC BY-NC-ND 4.0), which permits the noncommercial replication and distribution of the article with the strict proviso that no changes or edits are made and the original work is properly cited (including links to both the formal publication through the relevant DOI and the license). See: https://creativecommons.org/licenses/by-nc-nd/4.0/.

\section{References}

1. Kehlet H. Multimodal approach to control postoperative pathophysiology and rehabilitation. Br J Anaesth 1997;78:606-17.
2. Costelloe C, Burns S, Yong RJ, et al. An Analysis of Predictors of Persistent Postoperative Pain in Spine Surgery. Curr Pain Headache Rep 2020;24:11.

3. Coulthard P, Haywood D, Tai MA, et al. Treatment of postoperative pain in oral and maxillofacial surgery. Br J Oral Maxillofac Surg 2000;38:588-92.

4. Mehlisch DR, Aspley S, Daniels SE, et al. A singletablet fixed-dose combination of racemic ibuprofen/ paracetamol in the management of moderate to severe postoperative dental pain in adult and adolescent patients: a multicenter, two-stage, randomized, double-blind, parallel-group, placebo-controlled, factorial study. Clin Ther 2010;32:1033-49.

5. Evans SW, McCahon RA. Management of postoperative pain in maxillofacial surgery. Br J Oral Maxillofac Surg 2019;57:4-11.

6. Laine L. Approaches to nonsteroidal anti-inflammatory drug use in the high-risk patient. Gastroenterology 2001;120:594-606.

7. Fransen M, Anderson C, Douglas J, et al. Safety and efficacy of routine postoperative ibuprofen for pain and disability related to ectopic bone formation after hip replacement surgery (HIPAID): randomised controlled trial. BMJ 2006;333:519.

8. Chen XH, Bai JY, Shen F, et al. Imrecoxib: a novel and selective cyclooxygenase 2 inhibitor with anti-inflammatory effect. Acta Pharmacologica Sinica 2004;25:927-31.

9. Xu D, Zhang FC, Gu JR, et al. A multicenter double blind and randomized controlled phase III trail of imrecoxib in the treatment of knee osteroarthritis. Chinese Pharmaceutical Journal 2014;8:205-10.

10. Jia $X$, Long $M$, Huang $G$, et al. Effect of preemptive analgesia with imrecoxib on postoperative analgesic effect in patients undergoing spinal surgery. China Modern Doctor 2019;57:13-9.

11. Cheung R, Krishnaswami S, Kowalski K. Analgesic efficacy of celecoxib in postoperative oral surgery pain: a single-dose, two-center, randomized, double-blind, active- and placebo-controlled study. Clin Ther 2007;29 Suppl:2498-2510.

12. Beverly A, Kaye AD, Ljungqvist O, et al. Essential Elements of Multimodal Analgesia in Enhanced Recovery After Surgery (ERAS) Guidelines. Anesthesiol Clin 2017;35:e115-43.

13. Nimmo SM, Foo ITH, Paterson HM. Enhanced recovery after surgery: Pain management. J Surg Oncol 2017;116:583-91.

14. Isola G, Matarese M, Ramaglia L, et al. Evaluation of the 
efficacy of celecoxib and ibuprofen on postoperative pain, swelling, and mouth opening after surgical removal of impacted third molars: a randomized, controlled clinical trial. Int J Oral Maxillofac Surg 2019;48:1348-54.

15. Malmstrom K, Daniels S, Kotey P, et al. Comparison of rofecoxib and celecoxib, two cyclooxygenase-2 inhibitors, in postoperative dental pain: a randomized, placebo- and active-comparator-controlled clinical trial. Clin Ther 1999;21:1653-63.

16. Khan AA, Brahim JS, Rowan JS, et al. In vivo selectivity of a selective cyclooxygenase 2 inhibitor in the oral surgery

Cite this article as: Jiang Y, Qi L, Liu J, Wu H, Li Y, Zou L, Guo Z, Wang J, Li H. Analgesic efficacy of imrecoxib for postoperative pain following oral surgery: a prospective randomized, active-controlled, non-inferiority trial. Ann Transl Med 2021;9(6):469. doi: 10.21037/atm-21-264 model. Clin Pharmacol Ther 2002;72:44-9.

17. Gao GM, Li YM, Zheng XL, et al. The Efficacy of Imrecoxib and Celecoxib in Axial Spondyloarthritis and Their Influence on Serum Dickopff-Related Protein 1 (DKK-1) Levels. Med Sci Monit 2017;23:2985-92.

18. Zheng X, Zheng W, Zhang F. A multicenter openlabeled phase IV trial on imrecoxib in the treatment of Knee osteoarthritis. Chinese Journal of Rheumatology 2016;20:730-3.

(English Language Editor: A. Kassem) 